\title{
EDITORIAL
}

\section{LA INVESTIGACIÓN DE LAS ENFERMEDADES TRANSMISIBLES: UN ENFOQUE INTERDISCIPLINAR}

\author{
Ángela Domínguez $(1,2)$ \\ (1) Departamento de Salud Pública. Universidad de Barcelona. \\ (2) CIBER Epidemiología y Salud Pública. Instituto de Salud Carlos III.
}

A pesar de los grandes avances científicos y tecnológicos que se han producido en las últimas décadas en el campo de las infecciones que afectan a la humanidad, las enfermedades transmisibles, es decir aquellas infecciones que pueden trasmitirse al hombre por cualquier mecanismo, continúan siendo un problema de salud pública muy importante y nos ofrecen múltiples oportunidades para la investigación.

A finales de la década de los sesenta diversas personalidades del mundo de la salud pública, entre ellas el Surgeon General de los Estados Unidos, declararon que las enfermedades infecciosas habían sido conquistadas. La llegada del sida y la incidencia de nuevas infecciones puso de manifiesto que las enfermedades infecciosas constituyen un desafío constante ${ }^{1}$. Tanto la aparición de enfermedades causadas por agentes nuevos (SARS, gripe aviar, nueva gripe por el virus A (H1N1...) como la reemergencia a nivel global o en territorios definidos de enfermedades ya conocidas (tuberculosis, sarampión, rubeola, parotiditis, gastroenteritis por Norovirus) afectan o pueden afectar de manera importante el nivel de salud de las poblaciones de países desarrollados como el nuestro 2,3 . El momento histórico que vivimos se caracteriza por un importante desarrollo de los medios de transporte y comunicación y por un vertiginoso des- equilibrio económico mundial. Estos dos factores, coincidentes en el tiempo, propician un aumento de los viajes internacionales, tanto desde los países industrializados a los de baja renta (mediante el turismo, el comercio o la cooperación) como de éstos a los países industrializados (inmigración económica, adopciones internacionales, refugiados políticos... $)^{4-6}$. Ello conlleva que las enfermedades transmisibles en general y las enfermedades emergentes y reemergentes en particular supongan una preocupación constante para las autoridades de salud pública y que se generen importantes preguntas de investigación en relación a los determinantes de estas patologías.

En los últimos cinco años hemos observado en diversas comunidades autónomas que enfermedades que ya no se producían porque había dejado de circular el agente causal (el sarampión o la rubeola, por ejemplo) o que se producían en muy bajo número de casos (hepatitis A, por ejemplo) se han presentado en forma de brotes generados a partir de casos importados. Para algunas enfermedades, como la tuberculosis, la incidencia en la población inmigrante es mucho más elevada que en la población autóctona.

Paralelamente a estos fenómenos, la población inmigrante ha aumentado de 
manera muy importante en nuestro país, llegando a suponer el $15 \%$ de la población total en algunas comunidades y si bien los servicios sanitarios a los que tiene acceso dicha población son idénticos a los de la población autóctona, en la práctica se producen diferencias. Así, mientras que la cobertura de la primera dosis de vacuna triple vírica en Cataluña es del $98 \%$ en la población autóctona, sólo llega al $93 \%$ en la población inmigrante ${ }^{7}$. Estos fenómenos refuerzan la importancia de realizar encuestas de seroprevalencia que permitan identificar los grupos de población más susceptibles a determinadas enfermedades y que, por lo tanto, requieren mayor atención, así como también la de desarrollar modelos matemáticos que nos permitan predecir el comportamiento de determinadas enfermedades a partir de la experiencia previa ${ }^{8,9}$.

En este número monográfico de la Revista Española de Salud Pública, cuyo contenido científico he tenido la satisfacción de coordinar, se recogen los trabajos presentados en el Taller organizado por la Agrupación de Enfermedades Infecciosas y Salud Internacional del CIBER de Epidemiología y Salud Pública (Centro de Investigación Biomédica en Red: CIBERESP) del Instituto de Salud Carlos III, que tuvo lugar en Barcelona en junio de 2009, con el propósito de presentar y analizar diversas investigaciones sobre los cambios epidemiológicos ocurridos en determinadas enfermedades transmisibles de nuestro entorno. El taller, y también los trabajos que a continuación se presentan, tuvo un enfoque multidisciplinar, puesto que desde la Agrupación de Enfermedades Infecciosas y Salud Internacional entendimos que son diversas las disciplinas que convergen en la Salud Pública y que todas ellas deben tenerse en cuenta para orientar las estrategias preventivas adecuadamente. Si bien es cierto que la realidad de las enfermedades transmisibles es muy diversa y cambiante no es menos cierto que sólo aquello que podemos llegar a identificar y medir se puede incluir en las agendas de los responsables de Salud Pública y, por lo tanto, puede llegar a beneficiarse de actividades de prevención.

Las vacunas para las enfermedades inmunoprevenibles, el control de las personas enfermas y sus contactos en enfermedades como la tuberculosis o la enfermedad meningocócica, o las medidas de higiene de las manos, desinfección ambiental y aislamiento de los casos para las enfermedades de fácil transmisión fecal-oral, cuyo exponente más claro es la gastroenteritis por Norovirus, se pueden utilizar de manera más eficiente si se dispone de resultados de investigaciones epidemiológicas y de laboratorio. No cabe ninguna duda de que el conocimiento contribuye a las políticas de salud y que es la base que permite impulsar intervenciones efectivas y adecuar los servicios sanitarios a las necesidades de la población ${ }^{10}$.

De ahí la importancia de potenciar la investigación utilizando técnicas microbiológicas apropiadas para conocer la situación inmunitaria de la población frente a enfermedades emergentes o reemergentes y de impulsar entre los profesionales de los servicios de salud pública el desarrollo de métodos y técnicas matemáticas que nos permitan predecir el comportamiento de las enfermedades que en cada momento tengan más interés.

\section{BIBLIOGRAFÍA}

1. Detels R. Emerging infectious Diseases. En: Holland WW, Olsen J, Du Florey C, editors. The development of modern epidemiology. Oxford: Oxford University Press; 2008. 133-145.

2. Smolinsky MS, Hamburg MA,Ledergerg J, editors. Microbial threats to health. Washington: National Academic Press; 2003.

3. Ostoff SM, McDade JE, LeDuc JW, Hughes JM. Emerging and reemerging infectious disease threats. En: Mandell GL, Bennett JE, Dolin R, editors. 
Principles and Practice of Infectious Diseases. 6th ed. Philadelphia: Elsevier; 2005; 173-192.

4. White FMM, Nanan DJ. International and global health. En: Wallace RB, Kohatsu N, editors. New York: McGraw-Hill; 2007; 1251-7.

5. Reed CM, Steele S, Keystone JS. Health advice for international travel. En: Wallace RB, Kohatsu N, editors. New York: McGraw-Hill; 2007; 87-100.

6. Morens DM, Folkers Gk, Fauci As. The challenge of emerging and re-emerging infectious diseases. Nature. 2004; 430: 242-9.

7. Borrás E, Domínguez A, Batalla J, Torner N, Cardeñosa N, Nebot M, et al. Vaccination coverage in indigenous and immigrant children under 3 years of age in Catalonia (Spain). Vaccine. 2007; 25: 3240-3.

8. Anderson RM. Analytical theory of epidemics. En: Krause RM, editor. Emerging Infectious. San Diego: Academic Press; 2000; 23-50.

9. M Horsburgh CR, Mahon BE. Infectious disease epidemiology. En: Rothman KJ, Greenland S, Lash TL, editors. Modern Epidemiology. 3rd ed. Philadelphia: Wolters Kluwer; 2008; 549-63.

10. Ong A, Kindhauser M, Smith I, Chan M. Global health agenda for the twenty-first century. En: Detels R, Beaglehole R, Lansang MA, Gulliford M, editors. Oxford Textbook of Public Health. 5th ed.Oxford: Oxford University Press; 2009; 1713-29. 
